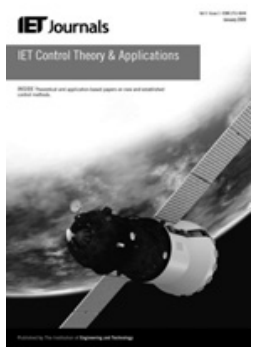

ISSN 1751-8644

\title{
Real-time estimation of tyre-road friction peak with optimal linear parameterisation
}

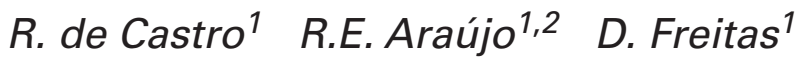 \\ ${ }^{1}$ Faculdade de Engenharia da Universidade do Porto, Rua Dr. Roberto Frias, 4200-465 Porto, Portugal \\ 2 INESCTEC (formerly INESC Porto), Rua Roberto Frias, 378 4200-465 Porto, Portugal \\ E-mail: de.castro@fe.up.pt
}

\begin{abstract}
Spurred by the problem of identifying, in real-time, the adhesion levels between the tyre and the road, a practical, linear parameterisation (LP) model is proposed to represent the tyre friction. Towards that aim, results from the theory of function approximation, together with optimisation techniques, are explored to approximate the non-linear Burckhardt model with a new LP representation. It is shown that, compared with other approximations described in the literature, the proposed LP model is more efficient, that is, it requires a smaller number of parameters, and provides better approximation capabilities. Next, a modified version of the recursive least squares, subject to a set of equality constraints on parameters, is employed to identify the LP in real time. The inclusion of these constraints, arising from the parametric relationships present when the tyre is in free-rolling mode, reduces the variance of the parametric estimation and improves the convergence of the identification algorithm, particularly in situations with low tyre slips. The simulation results obtained with the full-vehicle CarSim model under different road adhesion conditions demonstrate the effectiveness of the proposed LP and the robustness of the friction peak estimation method. Furthermore, the experimental tests, performed with an electric vehicle under low-grip roads, provide further validation of the accuracy and potential of the estimation technique.
\end{abstract}

\section{Introduction}

In automotive applications, the adhesion conditions present in the tyre-road interface have a strong influence in the tyre's ability to generate longitudinal and lateral forces and, under reduced grip conditions, represent a potential menace to the vehicle safety. With the recent proliferation of active safety systems (such as anti-lock braking system [ABS], traction control system [TCS], vehicle dynamics control [VDC]) [1], the estimation of adhesion levels, characterised by the friction coefficient, has attracted growing interest in the research community, since knowledge of this variable contributes significantly towards increasing the effectiveness of these safety systems. In addition, intelligent vehicles, as is the case with autonomous vehicles, currently at an early stage of development, can also benefit from the friction estimation, adapting the control strategies to the maximum grip levels available on the road [2]. Unlike other easily measurable variables, such as the vehicle acceleration, yaw rate and wheel speeds, there is currently no economically viable sensor that can be installed in the vehicle to measure the friction coefficient. These difficulties have encouraged the development of virtual sensors to estimate this variable using easily measurable signals.

There are several approaches to tackle the friction estimation problem. In this article, we focus on the so-called slip-based techniques, that is, the use of tyre force models based on the wheel slip to infer the adhesion levels [3], and constrain our study to the friction estimators active during longitudinal vehicle manoeuvres. These methods can be divided into two categories: qualitative and quantitative (see Fig. 1). In both cases, the main objective is to obtain an estimation of the peak friction coefficient; however, the outputs of the two mentioned methods are very different. In the first case, qualitative, the output of the estimator is based on a grading system, providing an indicator of adhesion quality, for instance, qualifying the grip levels on a scale from 'A' (high grip) to 'E' (very slippery); and in the second case, quantitative, a numerical output is generated to quantify the tyre-road adhesion in greater detail.

Examples of the qualitative methods can be found in [3-8]. The main driving force behind these approaches is the problem of persistence of excitation. To extract the peak friction, we need to apply high levels of tyre slip, which is not desirable from the safety point of view. To avoid this problem, the qualitative methods identify the tyre longitudinal stiffness, that is the friction slope for low-slip values, and then infer the grip levels through a classification process that correlates the slope value with the peak friction (see Fig. 1). Although these approaches solve the problem of persistence of excitation, they also introduce a new issue: the classification stage. This classification is very problematic to obtain in practice and, as pointed out by $[4,8,9]$, varies with the type of tyre, and the tyre wear, pressure and temperature, among many other factors. Therefore a significant experimental effort is required to tune the classification process, which makes these qualitative approaches very difficult to apply in practice. 


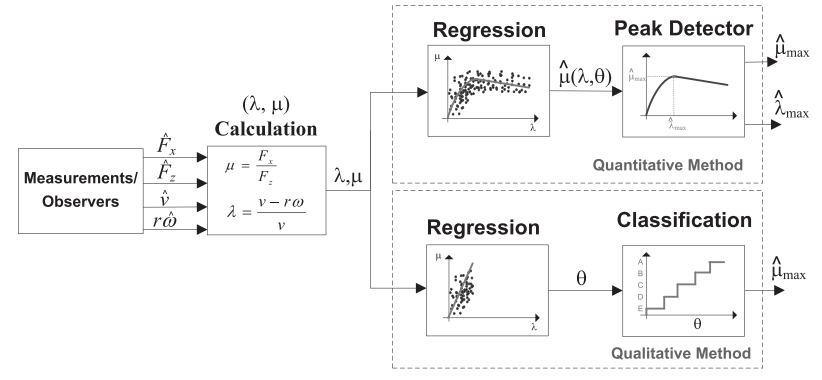

Fig. 1 Estimation methods for detecting the longitudinal peak friction

On the other hand, the quantitative methods [10-14] extract the peak friction using only online curve-fitting techniques. Although the estimation is obtained at the expense of applying high tyre slips, the estimation process is more simple and robust and also offers the possibility to identify the optimal slip reference $\lambda_{\max }$ associated with the maximum friction point. This last feature is very useful in generating the reference signal used in some anti-lock braking and traction control systems that rely on wheel slip control $[14,15]$. Furthermore, since the most common tyre models, such as the magic tyre formula (MTF) [16] or the Burckhardt representation [12], are non-linear, the online identification of the model parameters tends to be very difficult. As a result, most of the quantitative methods use linear parameterisation (LP), that is, non-linear models whose unknown parameters can be identified by linear techniques. Polynomials [11], rational functions [12, 17], exponentials [14], logistic sigmoids [18] and logarithmic functions $[13,19]$ are the most common LPs found in the literature.

In this article, we offer a contribution to the quantitative methods by proposing a more rational approach for deriving the LP. Unlike the aforementioned parameterisations, which are built using heuristics considerations, we employed optimal function approximation techniques to find an equivalent, but linear identifiable, model to the non-linear Burckhardt representation. The resulting LP yields a more accurate approximation and, in some cases, requires a smaller number of parameters, which gives some practical advantages for online friction identification. A second contribution to the quantitative framework is related to the application of a new and more robust identification technique to the peak friction estimation problem. Owing to its numeric efficiency and simplicity, the unconstrained recursive least squares (RLS) has become the most common method for estimating the friction parameters in real time $[12,14]$, but as shown in Section 4, under some parametric configurations, it may also introduce significant fitting error in the low-slip range and compromise the friction peak detection. To overcome this issue, we modify the RLS to comply with a set of (equality) constraints on the parameters, arising from the parametric relationships present when the tyre is in free-rolling mode. The inclusion of these constraints, which can be seen as a form of prior information on the system model, leads to a constrained RLS (CRLS) formulation and provides parameter estimates with less variance than the unconstrained version. Albeit the CRLS has been previously employed in other research fields (e.g. biomedical applications [20] and signal processing [21]), to the best of the authors' knowledge, no previous study has applied this technique to the problem of estimating the friction peak. Several simulations, performed with CarSim under different road adhesion conditions, demonstrate the higher accuracy and robustness of the CRLS against the RLS, particularly for the $\lambda_{\max }$ estimation. Finally, the proposed optimal LP and CRLS were also experimentally tested with an electric vehicle.

\section{Longitudinal vehicle/tyre model}

In this section, a brief introduction to the models related to the friction estimation methodology is provided. Since the longitudinal forces and slip are considered, the quarter car model, widely used in the literature on this field [12, 14, 22], is a sufficient mean for our study

$$
\begin{gathered}
J \dot{\omega}=r F_{x}-T_{\mathrm{b}} \\
M \dot{v}=-F_{x}
\end{gathered}
$$

where $\omega$ represents the wheel angular speed, $v$ the longitudinal vehicle speed, $T_{\mathrm{b}}$ the braking torque applied to the wheel, $F_{x}$ the friction force between tyre and the road, $J$ the wheel and transmission inertia, $M$ the equivalent mass coupled to the wheel and $r$ the wheel radius. For simplicity, in this work we only consider braking manoeuvres, hence $v \geq r \omega$, but the obtained results can be effortlessly modified for the acceleration case.

Modelling the longitudinal friction force $F_{x}$ is the main difficulty in (1). Generally, the friction force is proportional to the normal force that the wheel supports $\left(F_{z}\right)$ and depends on a non-linear function $\mu($.$) , known as the friction$ coefficient, which varies with the longitudinal tyre slip $(\lambda)$, road adhesion conditions, and tyre pressure, temperature, and wear, among other factors, and can be grouped into a parameter vector $\boldsymbol{\beta} \in \mathbb{R}^{d}$

$$
\begin{gathered}
F_{x}=F_{z} \mu(\lambda, \boldsymbol{\beta}) \\
\lambda=\frac{v-\omega r}{v}
\end{gathered}
$$

The most common approaches to represent the friction coefficient $\mu($.$) are based on two types of models: (i)$ static and (ii) dynamic. The static models, such as the Burckhardt [12] model

$$
\mu(\lambda, \boldsymbol{\beta})=\beta_{1}\left(1-e^{-\beta_{2} \lambda}\right)-\beta_{3} \lambda
$$

and the MTF [16]

$$
\mu(\lambda, \boldsymbol{\beta})=\beta_{1} \sin \left(\beta_{2} \operatorname{atan}\left(\left(1-\beta_{4}\right) \beta_{3} \lambda+\beta_{4} \operatorname{atan}\left(\beta_{3} \lambda\right)\right)\right)
$$

where the vector $\boldsymbol{\beta}=\left[\begin{array}{ll}\beta_{1} & \beta_{2}, \ldots, \beta_{d}\end{array}\right]^{\mathrm{T}}$ represents the model parameters, were developed by applying curvefitting techniques to the experimental tyre data. The great advantage of the static models is their simplicity; however, they are limited to steady-state conditions, and the model parameters lack physical meaning. More recently, dynamic models, such as the LuGre [22], have been applied to represent the tyre-road friction, presenting promising features to capture the friction transient behaviour. A complete review of friction models is beyond the scope of this work, but a detailed discussion about this topic can be found in $[22,23]$ (and references therein).

It is worth stressing the fact that the present work is only concerned with finding more effective LPs to improve 
the performance of the online regression stage depicted in Fig. 1. For this reason, we considered some practical assumptions, such as the access to the longitudinal and vertical tyre forces, vehicle velocity and wheel angular speed values (see Fig. 1), which is a common trend in the literature $[4,6,13,14]$. Of these variables, $\omega$ and $F_{z}$ are easily obtained. For example, the wheel speed is measurable with low-cost sensors, whereas the tyre vertical load can be extracted from well known weight transfer relations [12]. With regard to obtaining the longitudinal force information, there are two main possibilities: (i) direct measurement and (ii) observers. The former option can be accomplished through bearing units with load sensing [24, 25] or by putting accelerometers inside the tyre [26], but such sensors are generally costly and, as far as our knowledge goes, still remain largely in a prototype phase. To overcome the cost issue, longitudinal force observers represent an attractive prospect. For example, if the information about wheel torque is available, a simple force observer can be built from the model (1a), as discussed in $[12,27]$. Alternatively, the longitudinal vehicle dynamics (1b), together with the acceleration measurement, are also commonly employed to infer $F_{x}[3,6]$, whose performance can be further improved by applying the extended Kalman filter [28, 29]. Similarly to the longitudinal force information, the vehicle speed during braking can also be measured with dedicated sensors (e.g. with global positioning system (GPS) [3]) or estimated, for instance, using Kalman filtering [28] or fuzzy logic [12]. The measurement/observation of $F_{x}$ and $v$ will be revisited later in the article when we experimentally validate the estimation algorithm.

\section{Optimal LP}

In this section, we present the derivation of an optimal LP, in the sense that it provides the best fit of a non-linear friction model over a given parametric region of interest, as described in the next problem:

Problem 1 (linear approximation of $\mu$ ): Consider a model $f(\lambda, \boldsymbol{\beta})$, typically non-linear (such as (4) or (5))

$$
f: \mathcal{S} \times \mathcal{P} \rightarrow \mathbb{R}
$$

describing the friction coefficient curve in the domain $(\lambda, \boldsymbol{\beta}) \in \mathcal{S} \times \mathcal{P} \subset[0,1] \times \mathbb{R}^{d}$. The slip variable $\lambda$ is the model input, and the $\boldsymbol{\beta}$ vector contains the model internal parameters, which are unknown and possibly timevarying. To approximate this non-linear model, consider the parameterisation

$$
\begin{aligned}
\hat{f}(\lambda, \boldsymbol{w}, \boldsymbol{\theta}) & =\left[h_{1}(\lambda, \boldsymbol{w}), \ldots, h_{n}(\lambda, \boldsymbol{w})\right] \boldsymbol{\theta} \\
& =\boldsymbol{H}(\lambda, \boldsymbol{w})^{\mathrm{T}} \boldsymbol{\theta}
\end{aligned}
$$

where $\{\boldsymbol{w}, \boldsymbol{\theta}\} \in \mathbb{R}^{m} \times \mathbb{R}^{n}$ is a vector of parameters, and $h_{i}(\lambda, \boldsymbol{w}), i=1, \ldots, n$ are the basis functions. Under this setting, find the vector $\boldsymbol{w}^{*}$ that minimises the modelling error (a performance metric to express the notion of modelling error will be presented shortly) between $\hat{f}\left(\lambda, \boldsymbol{w}^{*}, \boldsymbol{\theta}\right)$ and $f(\lambda, \boldsymbol{\beta})$ over a given domain of interest $[0, \bar{\lambda}] \times \mathcal{D} \subset \mathcal{S} \times \mathcal{P}$.

Among the parameters $\{\boldsymbol{w}, \boldsymbol{\theta}\}$ of the approximator function, the $\boldsymbol{\theta}$ are easier to estimate, because of their linearity, whereas the $\boldsymbol{w}$ parameters have a non-linear effect in the model and complicate the identification process. Spurred by these difficulties, we investigate the possibility of selecting, offline, the best vector $\boldsymbol{w}^{*}$, in the sense that the fitting error is minimised, whereas $\boldsymbol{\theta}$ is estimated by online estimation methods in order to capture the variations of the unknown and time-varying internal parameters $(\beta)$ of (6). Notice that, after finding $\boldsymbol{w}^{*}$, we can insert this vector in (7) and transform the non-linear model into a LP, that is the model remains non-linear, but it is linear in the parameters $\boldsymbol{\theta}$. To keep the problem tractable, it is assumed that the number $(n)$ and type of basis functions are known beforehand (later we can evaluate the fitting performance for different numbers and types of basis functions).

At this stage, it is appropriate to discuss previous efforts towards solving Problem 1. Fixed basis functions (i.e. with $m=0$ ), such as polynomials [11] and rational functions [10], have been successfully employed for approximating the non-linear friction curve and have shown good performance in $\mu_{\max }$ peak friction estimation. However, as pointed out by de Castro et al. [18], the performance of these LPs deteriorates when $\lambda_{\max }$ estimations are needed. To overcome this difficulty, Tanelli et al. [14] proposed the use of exponential adaptive basis functions, which significantly boosts the $\mu_{\max }$ and $\lambda_{\max }$ estimation. Despite this, there is an important drawback with the LP proposed in [14]: a heuristic approach was used to select the coefficients $\boldsymbol{w}$ in the adaptive function, which does not generate the most efficient LP, that is, with a minimum number of basis functions. The present work extends these previous contributions by providing a systematic methodology to select the optimum value of the parameter $\boldsymbol{w}$, not only for exponentials but also for any type and number of basis functions.

The methodology presented in this article can be applied to various types of friction curves, but for simplicity, we use the Burckhardt parameterisation as a reference model and focus on finding an LP for the single non-linear term in this representation

$$
f(\lambda, \beta)=e^{-\beta \lambda}
$$

where $\lambda \in[0, \bar{\lambda}] \subset \mathbb{R}$ and $\beta \in \mathcal{D}=[\beta, \bar{\beta}] \subset \mathbb{R}$. For now, the offset and linear gain of (4) is omitted in the LP but will be included in the final parameterisation. According to [12], the parameter $\beta$ shows a strong dependence on the road conditions (e.g. dry tarmac, wet tarmac, snow etc.) and, to represent the most common types of roads, varies between $\beta=4$ and $\bar{\beta}=100$ (see Fig. 2). In addition, since the LP will be used to extract the friction peak, it is reasonable to assume that the LP should minimise the fitting error in the slip range between 0 and $\bar{\lambda}=0.5$ (note that it is uncommon to have friction peaks for longitudinal slips higher than $0.5[30])$.

Before solving the above-mentioned approximation problem, it is helpful to revisit a simplified dual problem: of the two vectors $\{\boldsymbol{w}, \boldsymbol{\theta}\}$ that parameterise the approximator (7), admit that $\boldsymbol{w}$ is known and consider the problem of finding the best set of linear parameters $\boldsymbol{\theta}$. If $\boldsymbol{\beta}$ is also known, we can then apply well-known results from the theory of function approximation to transform this problem into a minimum norm problem and extract $\boldsymbol{\theta}$ (see Appendix 1). The approach to solve Problem 1 proposed in the next section, builds on these analytical results but drops the assumption of known $\boldsymbol{w}$ (and $\boldsymbol{\beta}$ ). 


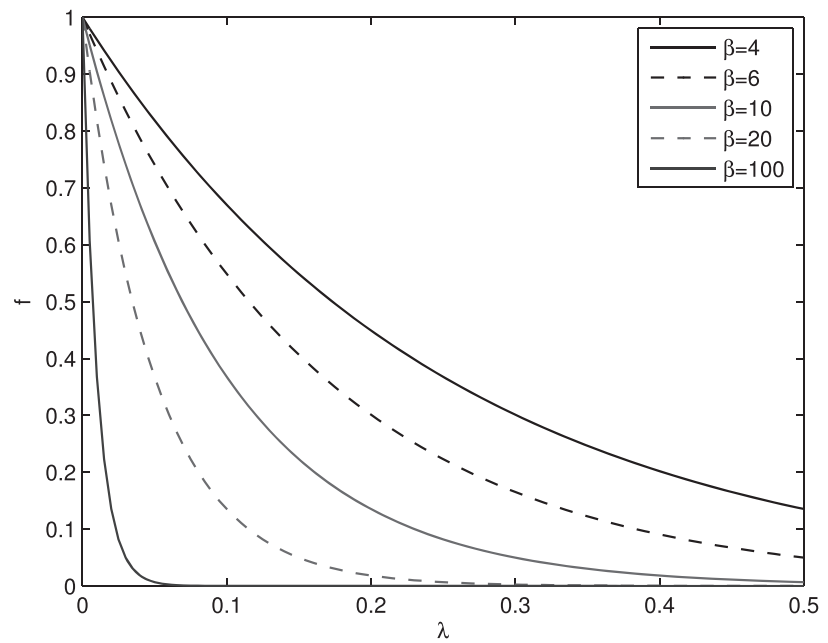

Fig. 2 Non-linear function $e^{-\beta \lambda}$, plotted for different values of $\beta$

\subsection{Optimal method for finding $L P$}

To tackle Problem (1), we start by defining the fitting error of the approximator ( 7$)$ for a given fixed triplet $(\beta, \boldsymbol{w}, \boldsymbol{\theta})$

$$
\varepsilon(\beta, \boldsymbol{w}, \boldsymbol{\theta})=\int_{0}^{\bar{\lambda}}(f(\lambda, \beta)-\underbrace{\boldsymbol{H}(\lambda, \boldsymbol{w})^{\mathrm{T}} \boldsymbol{\theta}}_{\hat{f}(\lambda, \boldsymbol{w}, \boldsymbol{\theta})})^{2} \mathrm{~d} \lambda
$$

Considering fixed values of $\beta$ and $\boldsymbol{w}$, the vector $\boldsymbol{\theta}$ that minimises $\varepsilon(\beta, \boldsymbol{w}, \boldsymbol{\theta})$ can be found by direct application of Lemma 1, presented in Appendix 1

$$
\begin{gathered}
\boldsymbol{\theta}(\beta, \boldsymbol{w})=\boldsymbol{G}^{-1}(\boldsymbol{w}) \boldsymbol{c}(\beta, \boldsymbol{w}) \\
{[\boldsymbol{G}(\boldsymbol{w})]_{i, j}=\left\langle h_{i}(\boldsymbol{w}), h_{j}(\boldsymbol{w})\right\rangle=\int_{0}^{\bar{\lambda}} h_{i}(\lambda, \boldsymbol{w}) h_{j}(\lambda, \boldsymbol{w}) \mathrm{d} \lambda} \\
{[\boldsymbol{c}(\beta, \boldsymbol{w})]_{i}=\left\langle f(\beta), h_{i}(\boldsymbol{w})\right\rangle \int_{0}^{\bar{\lambda}} f(\lambda, \boldsymbol{\beta}) h_{i}(\lambda, \boldsymbol{w}) \mathrm{d} \lambda}
\end{gathered}
$$

where $i, j=1, \ldots, n$. Replacing these relations in (9), the dependence on $\boldsymbol{\theta}$ can be eliminated and the fitting error redefined as

$$
\varepsilon(\beta, \boldsymbol{w})=\int_{0}^{\bar{\lambda}}\left(f(\lambda, \beta)-\boldsymbol{H}(\lambda, \boldsymbol{w})^{\mathrm{T}} \boldsymbol{G}^{-1}(\boldsymbol{w}) \boldsymbol{c}(\beta, \boldsymbol{w})\right)^{2} \mathrm{~d} \lambda
$$

Given that $\beta$ is known to belong to the set $\mathcal{D}$, it is reasonable to define a performance metric to sum all the fitting errors in this domain, which is obtained by integrating $\varepsilon(\beta, \boldsymbol{w})$ over $\mathcal{D}$. This new approximation metric, called total error, is defined by

$$
\varepsilon_{\mathrm{T}}(\boldsymbol{w})=\int_{\beta \in \mathcal{D}} \varepsilon(\beta, \boldsymbol{w}) \mathrm{d} \beta
$$

and depends only on $\boldsymbol{w}$. Thus, we can now pose an optimisation problem to extract the $\boldsymbol{w}$ parameter that minimises the total fitting error

$$
\begin{array}{ll}
\min _{\boldsymbol{w} \in \mathbb{R}^{m}} & \varepsilon_{\mathrm{T}}(\boldsymbol{w})=\min _{\boldsymbol{w} \in \mathbb{R}^{m}} \int_{\beta \in \mathcal{D}} \varepsilon(\beta, \boldsymbol{w}) \mathrm{d} \beta \\
\text { s.t. } & \text { eq.(11), (12), (13) }
\end{array}
$$

This problem assumes that the non-linear function $f(\lambda, \beta)$, the domain $[0, \bar{\lambda}] \times \mathcal{D}$, and the structure of the adaptive basis
Table 1 Total error $\varepsilon_{\mathrm{T}}$ for fitting (8) with different LPs

\begin{tabular}{lcccc}
\hline \multirow{2}{*}{ Basis type } & \multicolumn{4}{c}{ Number of basis $(n)$} \\
\cline { 2 - 5 } & 1 & 2 & 3 & 4 \\
\hline polynomial $\left(\boldsymbol{H}_{\mathrm{P}}\right)$ & - & 0.6844 & 0.3857 & 0.2127 \\
log. sigmoid $\left(\boldsymbol{H}_{\mathrm{L}}\right)$ & 0.2849 & 0.0467 & 0.0212 & 0.0059 \\
exponential $\left(\boldsymbol{H}_{\mathrm{E}}\right)$ & 0.2870 & 0.0362 & 0.0046 & 0.0005 \\
Tanelli et al. [14] & - & - & - & 0.0093 \\
\hline
\end{tabular}

functions $\boldsymbol{H}(\boldsymbol{\lambda}, \boldsymbol{w})$ are known, and delivers the parameter $\boldsymbol{w}$ that minimises the total fitting error, offering an optimal solution to Problem 1. Generally, the analytic treatment of the above problem is difficult, particularly when many basis functions are used. To overcome this issue, the integral (15) was discretised with a trapesoidal approximation and a numeric solver [31] employed to extract the optimal solution.

\subsection{LPs for approximating the Burckhardt model}

After presenting the methodology for deriving optimum LPs, we now evaluate the performance of different basis functions for approximating the single non-linear term (8) in the Burckhardt friction model. The approximating domain $[0, \bar{\lambda}] \times \mathcal{D}$ of the LP is the same as that discussed in the previous section, and the basis functions under consideration are

1. Polynomials: $\boldsymbol{H}_{\mathrm{P}}(\lambda)=\left[\begin{array}{lll}1 & \lambda & \lambda^{2}, \ldots, \lambda^{n-1}\end{array}\right]^{\mathrm{T}}$

2. Exponentials: $\boldsymbol{H}_{\mathrm{E}}(\lambda, \boldsymbol{w})=\left[e^{w_{1} \lambda}, \ldots, e^{w_{n} \lambda}\right]^{\mathrm{T}}$

3. Logistic sigmoid: $\boldsymbol{H}_{\mathrm{L}}(\lambda, \boldsymbol{w})=\left[\frac{1}{1+e^{-w_{1} \lambda-w_{2}}}, \ldots\right]^{\mathrm{T}}$

where $\boldsymbol{w}=\left[\begin{array}{ll}w_{1} & w_{2}, \ldots, w_{m}\end{array}\right]^{\mathrm{T}} \in \mathbb{R}^{m}$. Moreover, since we have a practical interest in minimising the LP complexity, different numbers of bases $(n \in\{1,2,3,4\})$ were also assessed.

A summary of the total error $\varepsilon_{\mathrm{T}}$ for the LPs under consideration can be found in Table 1. It is apparent from these results that the polynomial basis functions generate the worst fitting performance, and increasing $n$ does not significantly reduce the error. On the contrary, the approximation error decreases considerably by employing adaptive functions, and among these, the exponentials gives the best LP. For comparison purposes, the total fitting error obtained with the parameterisation defined in [14] is also provided: this LP produces a reasonable result, but it is clear that the total error can be decreased by using exponential basis functions, with optimum $\boldsymbol{w}$. Actually, we can even reduce the number of exponential functions to $n=3$ and still obtain total errors less than the ones produced by Tanelli et al. [14].

To provide additional insight regarding the performance of the LP, Fig. 3 shows the fitting error $\varepsilon$ over the approximating domain $\mathcal{D}$. Inspecting the polynomial performance reveals that the fitting errors increase, when $\beta$ gets higher; this was expected since, in this parametric region, the non-linear curve (8) starts to approach an impulse-like shape (see Fig. 2) and a high-order polynomial is needed to capture this behaviour. Furthermore, although the LP proposed in [14] is globally better than the polynomial, it still presents some significant errors for low $\beta$. These errors are further reduced with (optimal) exponential basis functions, and using $n=4$ basis generates the best LP over the entire domain $\mathcal{D}$. 


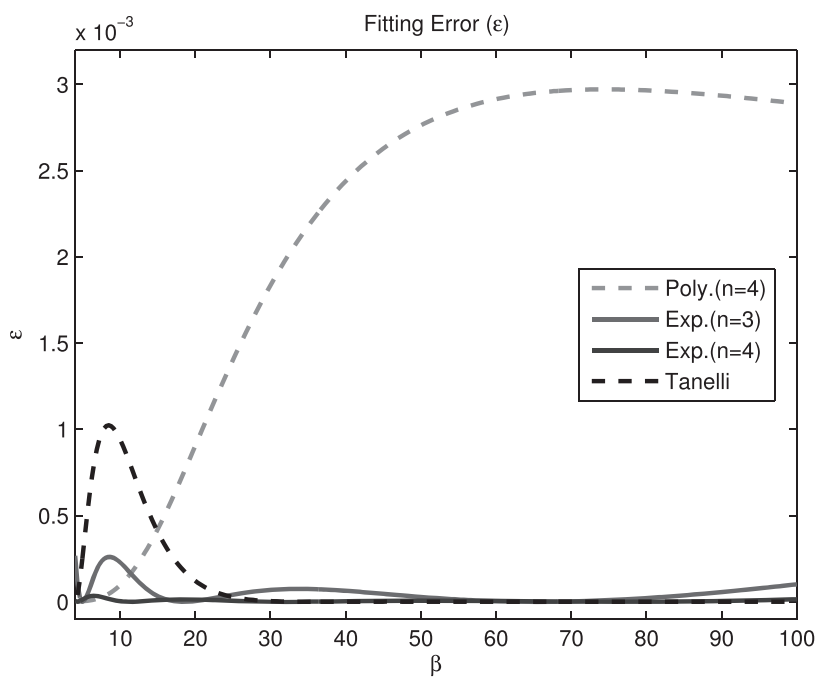

Fig. 3 Fitting error $\varepsilon(\beta)$ evaluated for polynomials, exponentials and the LP proposed in [14]

In summary, the optimal LP for approximating (8) was found to be exponential-based, with $n=4$ and $n=3$

$$
\begin{gathered}
\boldsymbol{H}_{\mathrm{E} 4}(\lambda)=\left[\begin{array}{llll}
e^{-4.28 \lambda} & e^{-11.37 \lambda} & e^{-32.34 \lambda} & e^{-77.05 \lambda}
\end{array}\right]^{\mathrm{T}} \\
\boldsymbol{H}_{\mathrm{E} 3}(\lambda)=\left[\begin{array}{lll}
e^{-4.99 \lambda} & e^{-18.43 \lambda} & e^{-65.62 \lambda}
\end{array}\right]^{\mathrm{T}}
\end{gathered}
$$

Based on this result, we can now join the linear terms of (4) with the LP of (8) and obtain the optimum LP that better approximates the Burckhardt friction model: $\hat{\mu}(\lambda, \boldsymbol{\theta})=\left[\begin{array}{lll}1 & \lambda & \boldsymbol{H}_{\mathrm{E}}(\lambda)^{\mathrm{T}}\end{array}\right] \boldsymbol{\theta}$. Although the performance of $\boldsymbol{H}_{\mathrm{E} 4}$ surpasses the other LPs, the basis $\boldsymbol{H}_{\mathrm{E} 3}$ is enough to provide a good fitting of the original non-linear model; on top of that, using $\boldsymbol{H}_{\mathrm{E} 3}$ enables us to eliminate one basis function, which may facilitate the online identification process. Accordingly, in the sequel, the LP

$$
\hat{\mu}(\lambda, \boldsymbol{\theta})=\boldsymbol{H}(\lambda)^{\mathrm{T}} \boldsymbol{\theta}=\left[\begin{array}{lll}
1 & \lambda & e^{-4.99 \lambda} e^{-18.43 \lambda} e^{-65.62 \lambda}
\end{array}\right] \boldsymbol{\theta}
$$

is used to approximate the Burckhardt friction model.

\subsection{Offline validation}

With the goal of validating the proposed LP, two 'offline' tests were conducted. The first is presented in Fig. $4 a$, which shows the performance of the LP defined in (18) against the original non-linear Burckhardt parameterisation [cf. (4)] for the most representative roads found in practice, as discussed in [12]. It can be noted that the LP and the Burckhardt curves are almost overlaid on each other, and little modelling error is introduced, thus providing additional evidence of the equivalence between both models.

An additional test was carried out to validate the LP with experimental data. This test is illustrated in Fig. $4 b$, which shows the LP curves after fitting with the classical least squares (LS) method, the experimental longitudinal force produced by a 205/55 R16 $90 \mathrm{H}$ tyre [32]. Once again, the fitting performance obtained with the LP was very satisfactory.

\section{Online estimation method}

After finding a suitable LP representation of the friction model in the tyre-road interface, we will discuss in this section a modified version of the RLS [33], capable of handling equality constraints, to identify the friction parameters. This method is known as the CRLS and is particularly useful in the current work to improve the accuracy and robustness of the friction estimation in the low-slip range.

\subsection{Motivation for CRLS}

To illustrate the relevance of the CRLS, consider two sets of input/output samples, $\mathcal{Z}=\{(\lambda(1), \mu(1)), \ldots,(\lambda(N), \mu(N))\}$, obtained from braking tests conducted in the CarSim simulator; the first set was performed on dry asphalt and the second on wet (see Fig. 5). The conditions under which these experiments were undertaken are described in the next section; for now, we merely discuss some of the potential pitfalls of the RLS. Owing to the recursive nature of the identification process, an initial guess for $\hat{\boldsymbol{\theta}}_{0}$ needs to be defined, which, following the initialisation procedure suggested in [14], was obtained by applying the traditional (batch) LS to the first collected
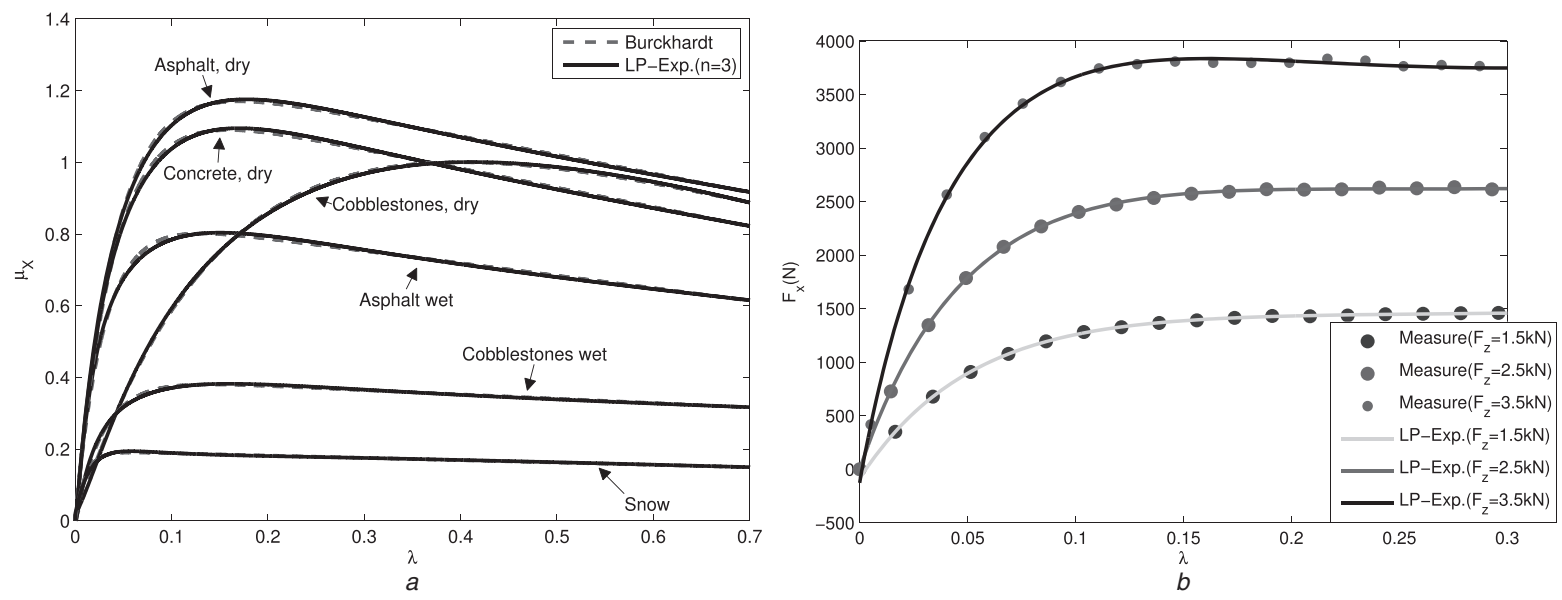

Fig. 4 Offline validation of the proposed LP

$a$ Comparison of the Burckhardt model [cf. (4)] with the LP proposed in this article [cf. (18)] for the most representative types of roads found in practice [12] $b$ Fitting of longitudinal force produced by a 205/55 R16 $90 \mathrm{H}$ tyre (experimental data were retrieved from [32]) using the LP (18) 

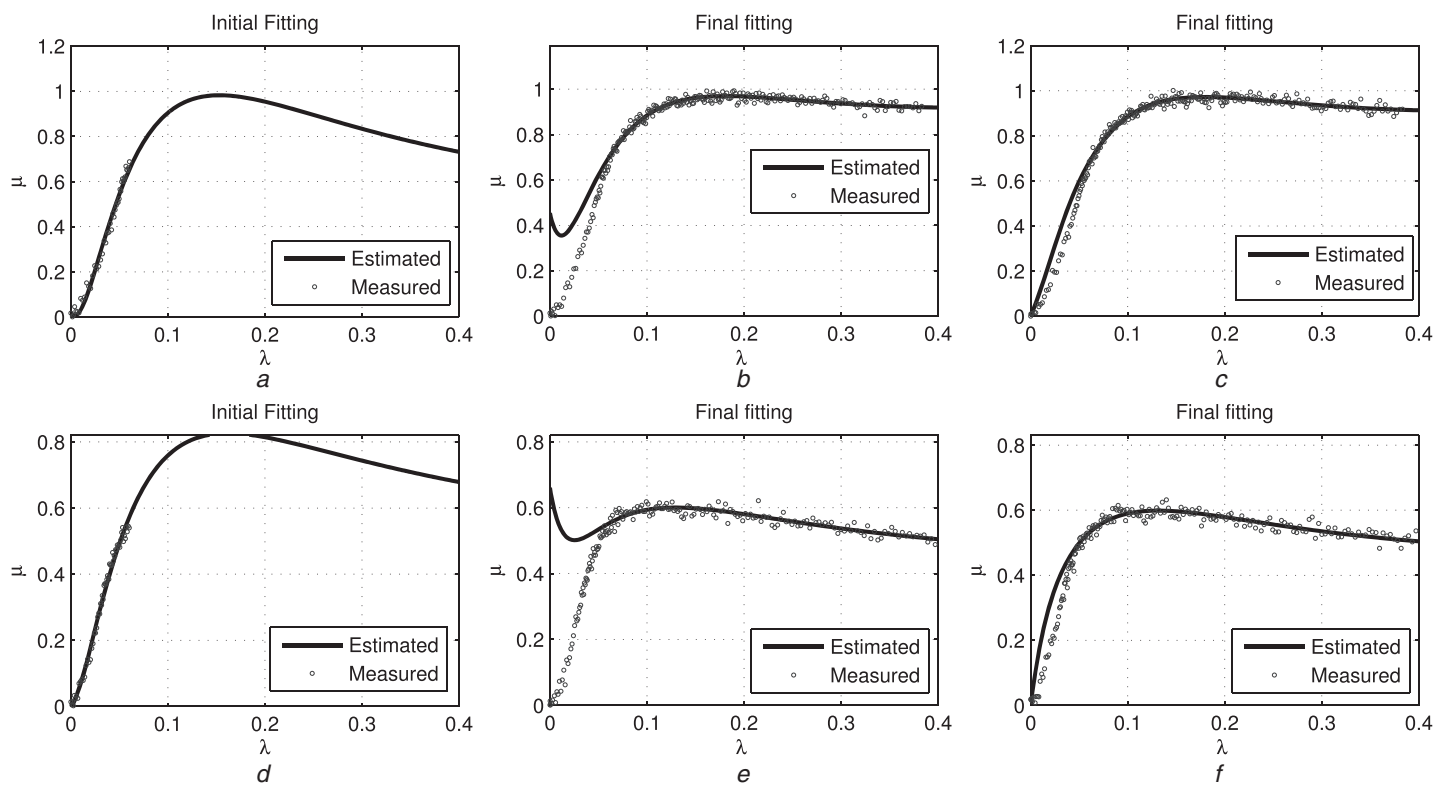

Fig. 5 Identification of the friction curve during braking on dry asphalt with $\mu_{\max }=1.0$ (top figures) and on wet asphalt with $\mu_{\max }=0.6$ (bottom figures)

$a$ and $d$ Initial estimate for the friction curve, calculated from the samples with $\lambda \leq \underline{\lambda}$

$b$ and $e$ Final friction curve estimate obtained after processing all the samples (see Fig. 6) with the RLS

$c$ and $f$ Final friction curve estimate obtained after processing all the samples (see Fig. 6) with the CRLS

samples, $\mathcal{Z}_{0}(\underline{\lambda})=\{(\lambda(k), \mu(k)) \in \mathcal{Z}: \lambda(k) \leq \underline{\lambda}\}$, where $\underline{\lambda}$ is the minimum threshold (0.06 in this work) to start the identification. After this initialisation step (Figs. $5 a$ and $b$ ), the remaining data in $\mathcal{Z}$ were processed with the RLS; the final fitting results are shown in Fig. $5 b$ for dry asphalt and in Fig. $5 e$ for wet asphalt. In the case of dry asphalt, it is clear that the RLS presents a very good fitting performance for tyre slips above 0.08 , but is prone to exhibit significant fitting errors in the low-slip range. Although this issue does not affect the detection of the friction peak in dry asphalt, when the RLS is evaluated in wet asphalt manoeuvres (Fig. 5e), the fitting error in the low-slip range increases and compromises the estimation process: the peak of the identified friction curve is located at zero slip, $\hat{\lambda}_{\max }=0$, whereas the true peak is around 0.12 . Accordingly, the peak friction estimation in wet asphalt has a significant error in the $\hat{\lambda}_{\max }$, which makes its use impractical for safety systems, such as the traction controller (TC) or the ABS. This problem was also observed in other low-friction conditions, but for the sake of brevity, these results were omitted in this section.

The most natural choice to attenuate this problem is to modify the RLS forgetting factor to increase the weight of the initial samples. Note, however, that the forgetting factor employed in these results is already relatively high $(\rho=0.999)$, thus little improvement is observed by modifying this parameter. Alternatively, if the initial estimate $\hat{\boldsymbol{\theta}}_{0}$ is good, which is far from guaranteed, we may try to reduce the parameter in the RLS associated with the confidence in the initial guess (see [33] and $\boldsymbol{P}_{0}$ in (28d)) and, as a consequence, decrease the amount of parameter variation during the recursive adaptation. Although they are possible, these parametric changes in the RLS tend to slow down the identification process, colliding with the need to quickly produce accurate estimations of the friction peak, which must be available before the vehicle safety systems become enabled.
This set of difficulties urged us to find ways of improving the RLS performance. A well-known possibility to increase the estimation performance consists of the use of prior information about the system model, for example, through a set of (equality and/or inequality) constraints on the model parameters [34-37]. In the current friction estimation problem, the prior information comes from the fact that the ideal friction curve must pass through the origin of the coordinate system $(\lambda, \mu)$ or, equivalently, comply with an equality constraint, $\boldsymbol{H}(0)^{\mathrm{T}} \boldsymbol{\theta}=0$. In other words, this constraint means that when the tyre is in free-rolling mode (i.e. $\lambda=0$ ), the friction force (and the instantaneous tyreroad friction coefficient $\mu$ ) should be zero. The remainder of this section will be devoted to explain how this equality constraint can be incorporated into the identification algorithm, called CRLS, and to highlight some of its theoretical and practical advantages.

\subsection{Description of the CRLS}

Just like the RLS, the starting point of the CRLS formulation is a weighted LS problem, but subject to an additional set of equality constraints

$$
\begin{aligned}
& \min _{\boldsymbol{\theta} \in \mathbb{R}^{n}} \sum_{k=1}^{t} \alpha(t, k)\left(\mu(k)-\mathbf{H}^{\mathrm{T}}(\lambda(k)) \boldsymbol{\theta}\right)^{2} \\
& \text { s.t. } \quad \mathbf{C} \boldsymbol{\theta}=\mathbf{d}
\end{aligned}
$$

where $t$ is the number of samples being considered, $\alpha($.) is the weight assigned to each sample and $C \in \mathbb{R}^{p \times n}$ and $\boldsymbol{d} \in \mathbb{R}^{p}$ characterise the set of $p$ linear constraints [it is assumed that $p<n$ and $\operatorname{rank}(\boldsymbol{C})=p$ ]. Reformulating the optimisation problem with the Lagrange multiplier, and taking into consideration the quadratic nature of the cost function, an analytical (batch) solution can be easily 
found $[34$, p. 225]

$$
\begin{gathered}
\hat{\boldsymbol{\theta}}_{t}=\boldsymbol{R}_{t}^{-1} \boldsymbol{f}_{t}+\boldsymbol{R}_{t}^{-1} \boldsymbol{C}^{\mathrm{T}}\left(\boldsymbol{C} \boldsymbol{R}_{t}^{-1} \boldsymbol{C}^{\mathrm{T}}\right)^{-1}\left(\boldsymbol{d}-\boldsymbol{C}_{t}^{-1} \boldsymbol{f}_{t}\right) \\
\mathbf{R}_{t}=\sum_{k=1}^{t} \alpha(t, k) \tilde{\boldsymbol{H}}(k) \tilde{\boldsymbol{H}}^{\mathrm{T}}(k) \\
\mathbf{f}_{t}=\sum_{k=1}^{t} \alpha(t, k) \tilde{\boldsymbol{H}}(k) \mu(k)
\end{gathered}
$$

where $\tilde{\boldsymbol{H}}(k)=\boldsymbol{H}(\lambda(k))$ is employed to simplify the notation.

Remark 1: From a convergence point of view, it is worth noting that, under some reasonable assumptions, the above batch estimator is unbiased and provides more precise results than the (unconstrained) LS. To prove these claims, we consider the following stochastic framework

$$
\mu(k)=\tilde{\boldsymbol{H}}^{\mathrm{T}}(k) \boldsymbol{\theta}+e(k)
$$

where $e(k)$ is the white noise measurement error, with mean $E[e]=0$ and variance $\sigma^{2}$. Further, it is assumed that $\{\tilde{\boldsymbol{H}}(k)\}$ and $\{e(k)\}$ are statistically independent, and, to simplify the mathematical treatment, a unitary weight $\alpha(t, k)=1$ is taken. Using the parameter estimation error

$$
\Delta \boldsymbol{\theta}=\hat{\boldsymbol{\theta}}_{t}-\boldsymbol{\theta}=\sum_{k=1}^{t}\left(\boldsymbol{I}-\gamma_{t} \boldsymbol{C}\right) \mathbf{R}_{t}^{-1} \tilde{\boldsymbol{H}}(k) e(k)
$$

where $\gamma_{t}=\boldsymbol{R}_{t}^{-1} \boldsymbol{C}^{\mathrm{T}}\left(\boldsymbol{C} \boldsymbol{R}_{t}^{-1} \boldsymbol{C}^{\mathrm{T}}\right)^{-1}$, we can determine the bias as

$$
\begin{aligned}
E[\Delta \boldsymbol{\theta}] & =\sum_{k=1}^{t} E\left[\left(\boldsymbol{I}-\gamma_{t} \boldsymbol{C}\right) \mathbf{R}_{t}^{-1} \tilde{\boldsymbol{H}}(k) e(k)\right] \\
& =\sum_{k=1}^{t} E\left[\left(\boldsymbol{I}-\gamma_{t} \boldsymbol{C}\right) \mathbf{R}_{t}^{-1} \tilde{\boldsymbol{H}}(k)\right] E[e(k)]
\end{aligned}
$$

Thus, the estimator will be unbiased, that is, $E[\Delta \boldsymbol{\theta}]=0$, if, besides the assumption of statistical independence, $E[e(k)]$ is zero, and $\mathbf{R}_{t}$ and $\left(\boldsymbol{C} \boldsymbol{R}_{t}^{-1} \boldsymbol{C}^{\mathrm{T}}\right)$ are non-singular. These last requirements represent the persistence of excitation conditions for the system. Next, consider the covariance of the parameter estimation error

$$
\begin{aligned}
\operatorname{cov}(\Delta \boldsymbol{\theta})= & E\left[\Delta \boldsymbol{\theta} \Delta \boldsymbol{\theta}^{\mathrm{T}}\right] \\
= & \sum_{k=1}^{t} \sum_{j=1}^{t} E\left[\left(\boldsymbol{I}-\gamma_{t} \boldsymbol{C}\right) \mathbf{R}_{t}^{-1} \tilde{\boldsymbol{H}}(k) e(k) e(j) \tilde{\boldsymbol{H}}(j)^{\mathrm{T}}\right. \\
& \left.\times \mathbf{R}_{t}^{-1}\left(\boldsymbol{I}-\gamma_{t} \boldsymbol{C}\right)^{\mathrm{T}}\right] \\
= & \sigma^{2} E\left[\mathbf{R}_{t}^{-1}\right]-\sigma^{2} E\left[\gamma_{t} \boldsymbol{C} \mathbf{R}_{t}^{-1}\right]
\end{aligned}
$$

and recall that the covariance matrix of the (unconstrained) LS is given by $\operatorname{cov}\left(\Delta \boldsymbol{\theta}^{\mathrm{LS}}\right)=\sigma^{2} E\left[\mathbf{R}_{t}^{-1}\right] \quad[35$, p. 236]. Replacing this relation in (26c), we obtain

$$
\operatorname{cov}\left(\Delta \boldsymbol{\theta}^{\mathrm{LS}}\right)-\operatorname{cov}(\Delta \boldsymbol{\theta})=\sigma^{2} E\left[\gamma_{t} \boldsymbol{C} \mathbf{R}_{t}^{-1}\right] \geq 0
$$

Therefore we can conclude that the constrained LS will be more efficient that the unconstrained LS, producing parameter estimates with smaller variance.
As this batch solution is of little use for online applications, it is necessary to find a recursive alternative. Following similar arguments to those in the RLS case, it is assumed that the weight function $\alpha(t, k)$ has the following properties [33]: (i) $\alpha(t, t)=1$ and (ii) $\alpha(t, k)=\rho \alpha(t-1, k)$, $0 \leq k \leq t-1$. These two properties can be satisfied using, for example, $\alpha(t, k)=\rho^{t-k}$, where $\rho$ is the forgetting factor. Based on these properties, and employing the well-known matrix inversion lemma, a recursive solution to $\boldsymbol{R}_{t}$ and $\boldsymbol{f}_{t}$ can be derived, which, after some algebraic manipulation, results in the following iterative solution of (20)

$$
\begin{gathered}
\hat{\boldsymbol{\theta}}_{t}=\hat{\boldsymbol{\theta}}_{t}^{\mathrm{RLS}}+\gamma_{t}\left(\mathbf{d}-\mathbf{C}_{t}^{\mathrm{RLS}}\right) \\
\hat{\boldsymbol{\theta}}_{t}^{\mathrm{RLS}}=\hat{\boldsymbol{\theta}}_{t-1}^{\mathrm{RLS}}+\boldsymbol{L}_{t}\left[\mu(t)-\tilde{\boldsymbol{H}}^{\mathrm{T}}(t) \hat{\boldsymbol{\theta}}_{t-1}^{\mathrm{RLS}}\right] \\
\boldsymbol{L}_{t}=\frac{\boldsymbol{P}_{t-1} \tilde{\boldsymbol{H}}(t)}{\rho+\tilde{\boldsymbol{H}}^{\mathrm{T}}(t) \boldsymbol{P}_{t-1} \tilde{\boldsymbol{H}}(t)} \\
\boldsymbol{P}_{t}=\frac{1}{\rho}\left[\begin{array}{c}
\left.\boldsymbol{P}_{t-1}-\frac{\boldsymbol{P}_{t-1} \tilde{\boldsymbol{H}}(t) \tilde{\boldsymbol{H}}^{\mathrm{T}}(t) \boldsymbol{P}_{t-1}}{\rho+\tilde{\boldsymbol{H}}^{\mathrm{T}}(t) \boldsymbol{P}_{t-1} \tilde{\boldsymbol{H}}(t)}\right] \\
\boldsymbol{\gamma}_{t}=\boldsymbol{P}_{t} \boldsymbol{C}^{\mathrm{T}}\left(\boldsymbol{C} \boldsymbol{P}_{t} \boldsymbol{C}^{\mathrm{T}}\right)^{-1}
\end{array}\right.
\end{gathered}
$$

It is interesting to note that the update equation (28a) of the recursive solution, is composed of two terms. The first $\left(\hat{\boldsymbol{\theta}}_{t}^{\mathrm{RLS}}\right)$ is in fact the solution to the unconstrained version of the problem, that is the traditional RLS, and because of this, (28b)-(28d) are the same as the RLS solution. The second term is a correction factor that ensures that the identified parameters respect the linear constraints in the iterations. Hence, from the numerical complexity point of view, the CRLS only demands an additional matrix equation (28e) to be evaluated, which, given the reduced number of unknown parameters (five) is not too penalising. Nonetheless, the calculation of the $\boldsymbol{\gamma}_{t}$ matrix involves an undesirable operation for online implementation: the inversion of the $\boldsymbol{C P}_{t} \boldsymbol{C}^{\mathrm{T}}$ matrix. To avoid this inversion, consider the gain $\boldsymbol{\gamma}_{t}$, rewritten as

$$
\boldsymbol{\gamma}_{t}=\boldsymbol{\Gamma}_{t} \boldsymbol{\eta}_{t}
$$

where $\boldsymbol{\Gamma}_{t}=\boldsymbol{P}_{t} \boldsymbol{C}^{\mathrm{T}}$ and $\boldsymbol{\eta}_{t}=\left(\boldsymbol{C} \boldsymbol{\Gamma}_{t}\right)^{-1}$. Following the algebraic manipulation suggested in [21], a recursive version of $\boldsymbol{\Gamma}_{k}$ can be established by multiplying (28d) with $\boldsymbol{C}^{\mathrm{T}}$, whereas the matrix inversion lemma is again applied to calculate $\eta_{k}$ iteratively

$$
\begin{gathered}
\boldsymbol{\Gamma}_{t}=\frac{1}{\rho}\left(\boldsymbol{\Gamma}_{t-1}-\boldsymbol{L}_{t} \tilde{\mathbf{H}}^{\mathrm{T}}(k) \boldsymbol{\Gamma}_{t-1}\right) \\
\boldsymbol{\eta}_{t}=\rho\left(\boldsymbol{\eta}_{t-1}+\frac{\boldsymbol{\eta}_{t-1} \boldsymbol{C} \boldsymbol{L}_{t} \tilde{\mathbf{H}}^{\mathrm{T}}(k) \boldsymbol{\Gamma}_{t-1} \boldsymbol{\eta}_{t-1}}{1-\tilde{\mathbf{H}}^{\mathrm{T}}(k) \boldsymbol{\Gamma}_{t-1} \boldsymbol{\eta}_{t-1} \boldsymbol{C} \boldsymbol{L}_{t}}\right)
\end{gathered}
$$

Therefore the final, numeric efficient, CRLS algorithm used in this work is described by (28a)-(28d), (29) and (30). Similar to the RLS, the CRLS also needs an initial guess for $\hat{\boldsymbol{\theta}}_{0}$ and $\boldsymbol{P}_{0}$, which are then used to initialise the remaining recursive variables $\left(\boldsymbol{\Gamma}_{0}=\boldsymbol{P}_{0} \boldsymbol{C}^{\mathrm{T}}\right.$ and $\left.\boldsymbol{\eta}_{0}=\left(\boldsymbol{C} \boldsymbol{\Gamma}_{0}\right)^{-1}\right)$.

\subsection{CRLS fitting performance}

Returning to the friction estimation, the CRLS was applied taking into account the single restriction in the problem

$$
\boldsymbol{H}(0)^{\mathrm{T}} \boldsymbol{\theta}=\underbrace{\left[\begin{array}{lllll}
1 & 0 & 1 & 1 & 1
\end{array}\right]}_{\boldsymbol{C}} \boldsymbol{\theta}=\underbrace{0}_{\boldsymbol{d}}
$$


The CRLS performance, presented in Figs. $5 c$ and $f$, shows, as expected, that the identified friction curve always passes through the origin, which contributes to a much better fitting in the low-slip range, both on dry and wet asphalt. As will be shown in the next section, this CRLS feature is key for improving the identification robustness against the regression method parameters, for example, the forgetting factor and the initial estimate, and extracts a precise estimation of the peak friction, particularly in low-grip conditions.

\section{Simulation results}

To evaluate the performance of the peak friction estimation with the optimal LP and the CRLS identification method, this section presents the simulation results obtained with the CarSim simulator [38]. The estimation framework used is a quantitative one and, as shown in Fig. 1, can be divided into three steps: (i) collection of $\lambda$ and $\mu$ samples, (ii) fitting/regression and (iii) peak detector. These steps were implemented as follows:

1. Regarding the first stage, it was assumed that the estimation algorithm has access to the samples $\mu(k)$ and $\lambda(k)$, where $k$ is the sampling instant. In simulation tests, these variables are easily available.

2. For the regression step, the friction model adopted was the LP proposed in Section 3 [cf. (18)], which was used to evaluate two types of identification methods: the traditional RLS and the CRLS. To make a fair comparison, the algorithms had the same initial parameters, that is forgetting factor $(\alpha=0.999) ; \boldsymbol{P}_{0}=\delta \boldsymbol{I}$, where $\delta=100$ and $\boldsymbol{I}$ is the identity matrix; and initial guesses $\hat{\boldsymbol{\theta}}_{0} \in \mathbb{R}^{n}$ (which were obtained by the method suggested in [14] and described in Section 4.1).

3. The final step in the algorithm was obtained by computing the friction maximum point

$$
\begin{gathered}
\hat{\mu}_{\max }=\max _{\lambda} \hat{\mu}(\lambda, \hat{\boldsymbol{\theta}}) \\
\hat{\lambda}_{\max }=\underset{\lambda}{\arg \max } \hat{\mu}(\lambda, \hat{\boldsymbol{\theta}})
\end{gathered}
$$

The identification algorithm presented above was evaluated using a typical A-class hatchback car, available in the CarSim library, having 175/70 R13 tyres, modelled with the MTF 5.2 [16]. The MTF 5.2 parameters are omitted here because of their high number (more than 80 ); in any case, the model reflects the steady-state behaviour of a real tyre and takes into account some simple dynamic transients, such as the relaxation length. The sampling time for the algorithm was set to $2 \mathrm{~ms}$ and, to contemplate the measuring errors that normally appear in this application, the variable $\mu$ was corrupted with Gaussian noise, with variance $\sigma_{\mu}^{2}=0.015^{2}$.

Based on these settings, three types of braking manoeuvres were performed in straight line, under different grip conditions: dry asphalt, wet asphalt and snow. The input data generated by these manoeuvres are shown on the left part of Fig. 6, whereas the estimation results are on the right. Analysing the estimations $\hat{\lambda}_{\max }$ and $\hat{\mu}_{\max }$ for all the tests, it can be observed that no value is generated in the first $\sim 0.1 \mathrm{~s}$; during this period of time, the estimator is disabled because the slip is below the threshold activation point $\underline{\lambda}$, and these initial samples are used to calculate an initial guess for $\hat{\boldsymbol{\theta}}_{0}$. Inspecting the peak friction estimation for dry
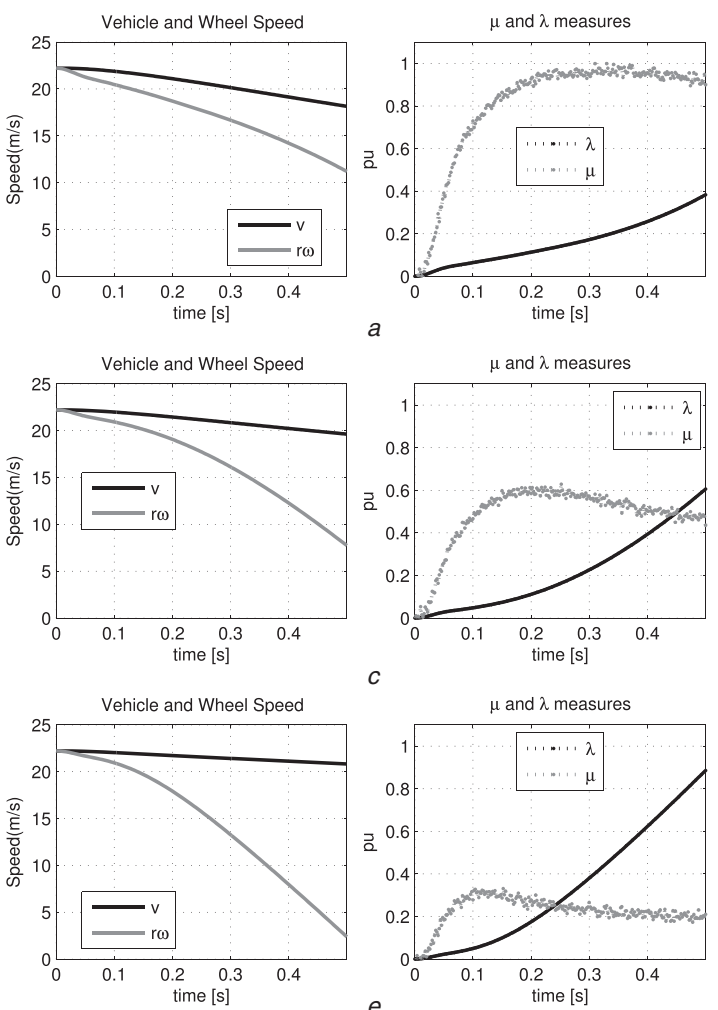

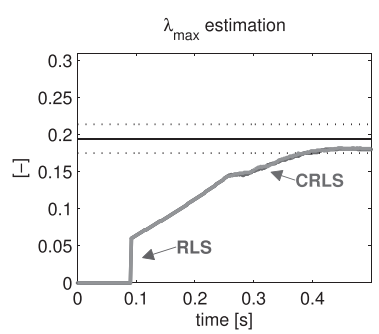

$\lambda_{\max }$ estimation

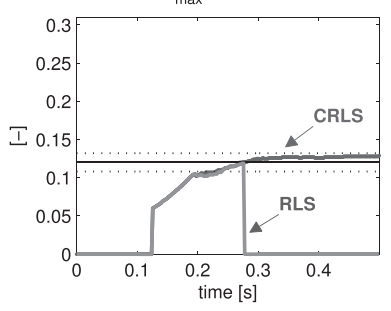

$\lambda_{\text {max }}$ estimation

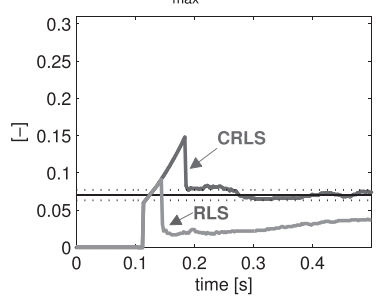

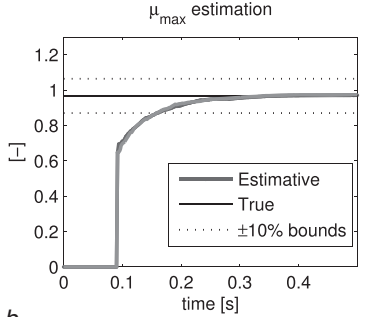

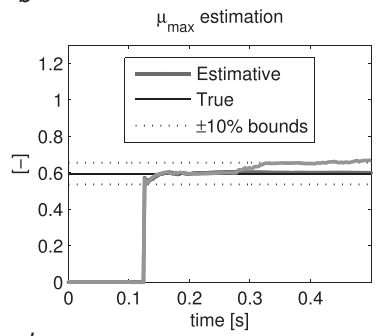

$d$

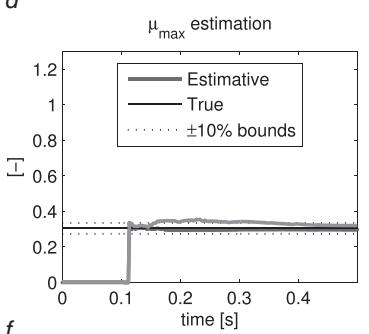

Fig. 6 Simulation results with the CarSim for different types of road adhesion 
asphalt, shown in Fig. 6b, one can find that the RLS and the CRLS present an almost equal performances and converge, in less than $0.4 \mathrm{~s}$, to the proximities of the true values of $\mu_{\max }$ and $\lambda_{\max }$. This similar behaviour can be explained by the fact that the true friction peak is moderately high $\left(\lambda_{\max } \simeq 0.2\right.$ ), whereas the fitting error introduced by the RLS (see Fig. $5 b$ ) mainly affects the low-slip regime $(\lambda \leq 0.08)$. On the other hand, when the manoeuvre is performed on wet asphalt (Fig. $6 d$ ), the $\hat{\lambda}_{\max }$ estimation obtained with the RLS shows a severe estimation error after $0.3 \mathrm{~s}$, whereas the CRLS maintains a very satisfactory performance. Unlike in the previous case (dry), on wet asphalt the $\lambda_{\max }$ is relatively low $(\sim 0.12)$, and the fitting error introduced by the RLS (see Fig. 5e) is sufficiently high to compromise the peak friction estimation. The snow test (Fig. $6 f$ ) also shows a significant estimation error in $\hat{\lambda}_{\max }$, albeit less severe than on wet asphalt. It is also worth mentioning that the $\hat{\mu}_{\max }$ estimation is less sensitive to the fitting errors in low slip, and for that reason, both the RLS and the CRLS show similar performances in $\hat{\mu}_{\max }$ in all the adhesion conditions under test.

In conclusion, these simulation results highlight the robustness of the CRLS over the traditional RLS in the peak friction estimation. Moreover, we can also conclude that by employing the CRLS, the designer has more freedom to select the forgetting factor $(\rho)$ and decrease the confidence $\left(\boldsymbol{P}_{0}\right)$ in the initial guess without fear of the fitting problems in the low-slip region that appear in the RLS and mainly affects the $\lambda_{\max }$ estimation in low-grip roads.

\section{Experimental results}

The peak friction estimator presented in the previous sections was experimentally validated in a neighbourhood electric vehicle, which belongs to a class of low-speed vehicles suitable for urban mobility whose main feature is a decoupled powertrain structure with two electric motors individually connected to the front wheels (Fig. 7). The field-programmable gate array (FPGA) XC3S1000 was used to implement the main powertrain controller functions; it has three important properties: multi-motor control [39], minimisation of motor energy losses [40] and wheel slip control [41]. The peak friction estimator is an additional functionality fundamental for generating the ideal slip reference for the wheel slip controller, which is currently being incorporated into the powertrain controller (Fig. $7 d$ ). Nevertheless, in this work we are only concerned with the development and validation of the friction peak estimator, and for this reason, we leave the integration issues with the wheel slip control to future endeavours.

The implementation of the peak friction estimator again followed the three steps described in the previous section but with some important modifications. First, although in

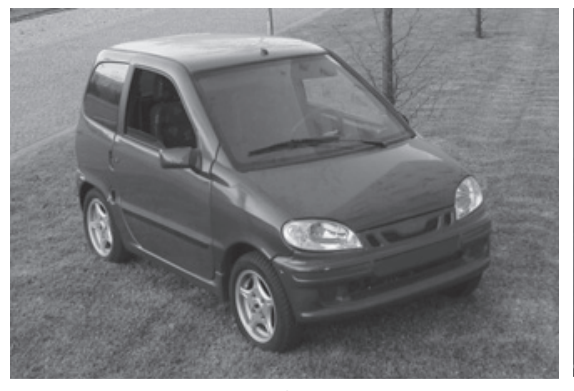

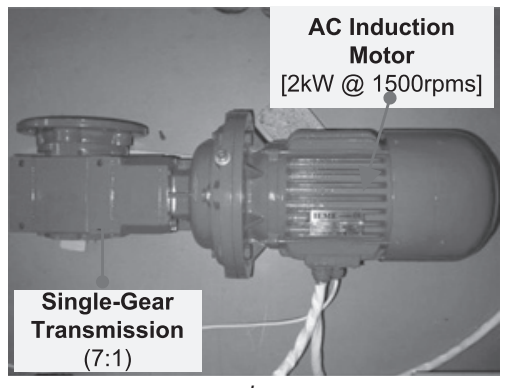

$b$

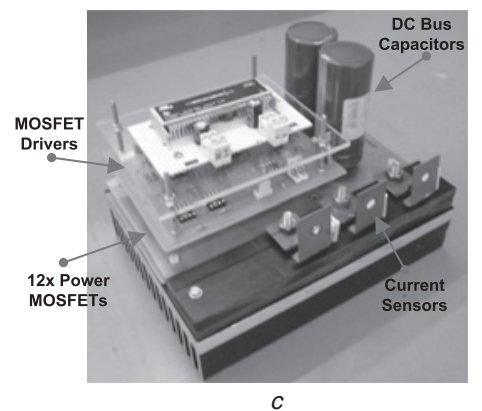

$c$

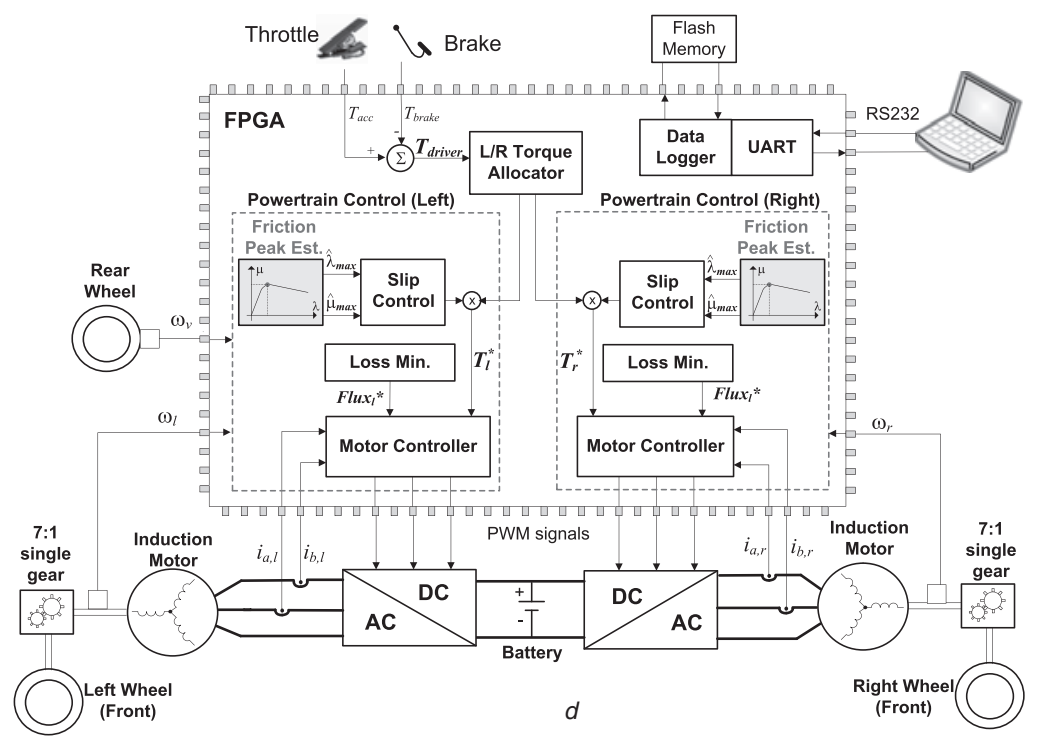

Fig. 7 Electric vehicle, powertrain, and control structure employed during the experimental validation of the peak friction estimator $a$ Vehicle

$b$ Powertrain of each front wheel

$c$ DC/AC power converter

$d$ Block diagram of the powertrain control system 


\section{www.ietdl.org}

the simulations the $\mu$ measures are easily obtained, in the experimental validation, such information must be estimated. Consequently, the $\mu$ samples were extracted from the quarter car model (1a), (2)

$$
\mu=\frac{T_{\mathrm{b}}}{r F_{z}}-\frac{J}{r F_{z}}\left(\frac{s}{\tau_{f} s+1}\right) \omega
$$

where $s$ is the Laplace operator. The model parameters are the wheel radius $(r=0.26 \mathrm{~m})$ and the wheel inertia $\left(J=0.6 \mathrm{~kg} \mathrm{~m}^{2}\right)$, which are assumed constant throughout the experimental tests. To avoid the noise issues with the differentiation, this operator was approximated with a high-pass filter, having a time constant of $\tau_{f}=40 \mathrm{~ms}$ (this approximation is also known as the 'dirty differentiation' [42]), and discretised with the bilinear transformation. The vertical load supported by the wheel $\left(F_{z}\right)$ can be extracted from simple weight transfer relations [12], whereas the braking torque is easily inferred from the motor current measures. The wheel slip samples $\lambda$ were calculated using (3) and the vehicle speed was obtained from the non-driven wheels (note that during the experimental tests, only the electric vehicle (EV) front wheels were driven).

A second factor to be taken in consideration is the initial guess for the parameters $\hat{\boldsymbol{\theta}}_{0}$; two types of initial guesses were assessed:

1. Initialisation A: the Burckhardt dry curve (cf. Fig. $4 a$ ) is used as the initial guess of $\hat{\boldsymbol{\theta}}_{0}$

$$
\hat{\boldsymbol{\theta}}_{0}=\left[\begin{array}{lllll}
1.22 & -0.45 & 0.18 & -1.19 & -0.25
\end{array}\right]^{\mathrm{T}}
$$

2. Initialisation $\mathrm{B}: \hat{\boldsymbol{\theta}}_{0}$ is obtained following the procedure suggested in [14], used in the simulations and described in Section 4.1.

Although the second option may give more accurate (initial) estimates and contribute to faster convergence, from the implementation point of view, it requires higher computational effort since the batch LS must be executed before the RLS. In the simulation and experimental tests performed in this work, we noticed that, depending on the manoeuvre, the size of the initial set of samples that the LS must handle (see the set $\mathcal{Z}_{0}$ in Section 4.1) can vary from 20 to 30 samples, which, for real-time applications with limited computational resources, may pose a serious computational burden. On the other hand, initialisation A does not require any extra calculations, but there are some doubts about the algorithm convergence speed under low-grip conditions. To dissipate such doubts, the performances of these two initialisations are assessed in the experimental tests. Moreover, since the superiority of the CRLS has been established in the simulations, only this identification method is implemented (with the same configurations and sampling time as the ones used in the previous sections).

Fig. 8 shows the acquired data on the left wheel during a braking operation performed on a low-grip surface, as well as the peak friction estimation, obtained with the slip control disabled. Before the driver applies a torque, both estimators are disabled because the slip does not reach the minimum threshold $\underline{\lambda}$, and for these reasons, initialisation A outputs a nominal dry estimate, whereas initialisation B does not provide any estimate. At around $21 \mathrm{~s}$, a significant braking
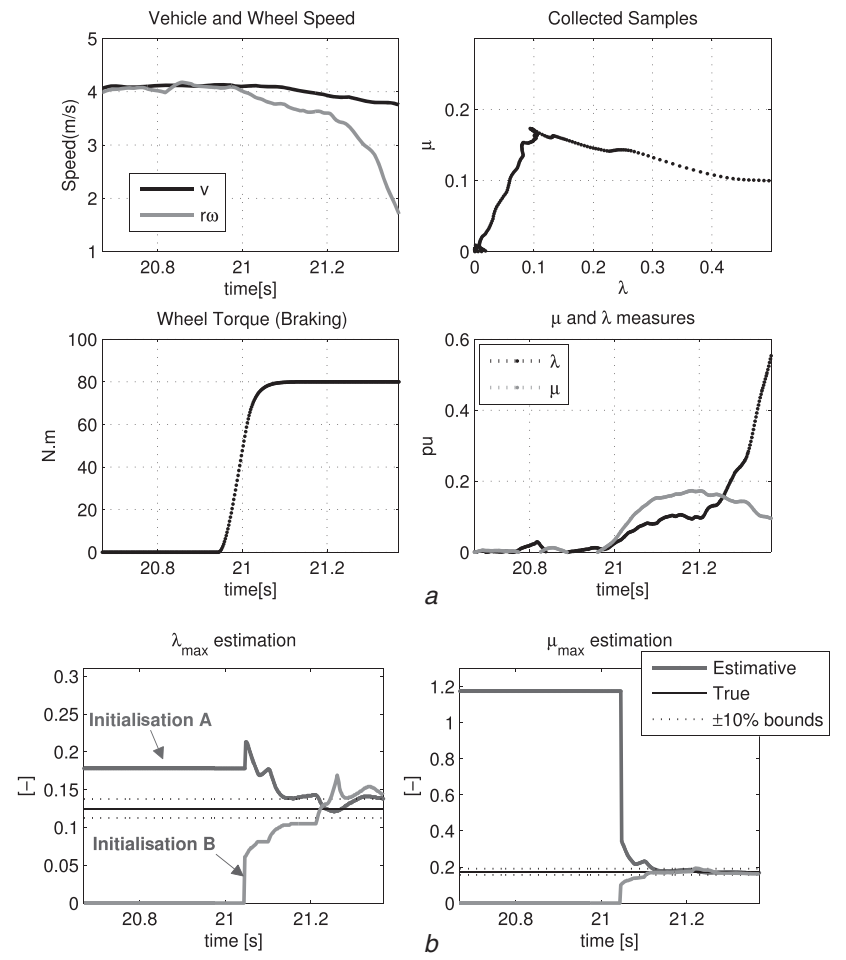

Fig. 8 Experimental results for the left wheel during a braking manoeuvre on a low-grip surface

a Acquired data

$b$ Peak friction estimation with CRLS

torque is applied, which enables the CRLS adaptation, and after $0.3 \mathrm{~s}$ of iterations, both initialisations produce satisfactory results in the $\hat{\mu}_{\max }$ estimation. Regarding $\hat{\lambda}_{\max }$, it is interesting to point out the following: (i) during the initial transient, initialisation A has a tendency to overestimate the peak friction, whereas initialisation $\mathrm{B}$ is more prone to underestimation, which are expected given the $\hat{\boldsymbol{\theta}}_{0}$ employed in each case (this observation also applies to $\hat{\mu}_{\max }$ ); and (ii) initialisation A provides a slightly more accurate final estimation of $\hat{\lambda}_{\max }$ and, after $21.1 \mathrm{~s}$, stays inside the predefined tolerance band of $\pm 10 \%$, whereas initialisation $B$ shows a slightly larger estimation error. Hence, based on this second observation, we can state that although initialisation $B$ provides a better initial fitting for the slip range below $\underline{\lambda}$, this is no guarantee that the (final) identified friction curve will generate a better peak friction estimate. Moreover, this challenging experimental test highlights that the most computation-efficient initialisation (A), together with the optimal LP and the CRLS, is sufficient to generate good peak friction estimation without penalising the convergence speed or final accuracy.

\section{Conclusions}

In this work, a systematic methodology to extract an optimal LP to represent the tyre-road friction was developed. Towards this aim, we exploited the fact that the structure of the non-linear friction model is known beforehand, such as the Burckhardt representation, and applied analytic, as well as numeric, optimisation techniques to extract the LP with minimum fitting error along a given parametric range of interest. It was shown that the modelling error introduced by the optimum LP is almost negligible and outperforms 
others LPs previously proposed in the literature. The linear structure featured by the LP simplifies the real-time friction identification process, since we can rely on linear regression methods to accomplish this task. A constrained version of the RLS was then employed to further increase the robustness of the peak friction identification against initial errors in the (linear) parameters and the RLS tuning parameters, for example, the forgetting factor. Simulation results, obtained with the CarSim simulator, showed that the optimal LP identified with the CRLS provides a satisfactory performance in the estimation of $\hat{\lambda}_{\max }$ and $\hat{\mu}_{\max }$ under different tyre-road adhesion levels. These results were further validated with experimental tests, obtained with a neighbourhood electric vehicle on a low-grip surface.

As future endeavours, we plan to extend the experimental work to address different types of surfaces, and study further extensions of the LP to handle situations with lateral and combined tyre slip.

\section{Acknowledgment}

This work was partially funded by FCT - Science and Technology Foundation, through the scholarship number SFRH/BD/47882/2008, and by MCA \& Associados, Lda.

\section{References}

1 van Zanten, A.T.: 'Evolution of electronic control systems for improving the vehicle dynamic behavior'. Proc. Int. Symp. on Advanced Vehicle Control (AVEC), Hiroshima, Japan, 2002, pp. 7-15

2 Talvala, K.L.R., Kritayakirana, K., Gerdes, J.C.: 'Pushing the limits: from lanekeeping to autonomous racing', Annu. Rev. Control, 2011, 35, (1), pp. 137-148

3 Rajamani, R., Piyabongkarn, N., Lew, J., Yi, K., Phanomchoeng, G.: 'Tire-road friction-coefficient estimation', IEEE Control Syst. Mag., 2010, 30, (4), pp. 54-69

4 Gustafsson, F.: 'Slip-based tire road friction estimation', Automatica, 1997, 33, (6), pp. 1087-1099

5 Ono, E., Asano, K., Sugai, M., Ito, S., Yamamoto, M., Sawada, M., Yasui, Y.: 'Estimation of automotive tire force characteristics using wheel velocity', Control Eng. Pract., 2003, 11, (12), pp. 1361-1370

6 Muller, S., Uchanski, M., Hedrick, K.: 'Estimation of the maximum tire-road friction coefficient', J. Dyn. Syst., Meas. Control, 2003, 125, (4), pp. 607-617

7 Wang, J.M., Alexander, L., Rajamani, R.: 'Friction estimation on highway vehicles using longitudinal measurements', J. Dyn. Syst. Meas. Control-Trans. ASME, 2004, 126, (2), pp. 265-275

8 Andrieux, A., Vandanjon, P.O., Lengelle, R., Chabanon, C.: 'New results on the relation between tyre-road longitudinal stiffness and maximum available grip for motor car', Veh. Syst. Dyn., 2010, 48, (12), pp. 1511-1533

9 Carlson, C.R., Gerdes, J.C.: 'Consistent nonlinear estimation of longitudinal tire stiffness and effective radius', IEEE Trans. Control Syst. Technol., 2005, 13, (6), pp. 1010-1020

10 Kiencke, U.: 'Realtime estimation of adhesion characteristic between tyres and road'. Proc. IFAC 12th Triennial World Congress, 1993, 1, pp. $15-22$

11 Germann, S., Wurtenberger, M., Daiss, A.: 'Monitoring of the friction coefficient between tyre and road surface'. Proc. Third IEEE Conf. on Control Appl., 1994, 1, pp. 613-618.

12 Kiencke, U., Nielsen, L.: 'Automotive control systems for engine, driveline, and vehicle' (Springer-Verlag, 2005)

13 Yi, J., Alvarez, L., Horowitz, R.: 'Adaptive emergency braking control with underestimation of friction coefficient', IEEE Trans. Control Syst. Technol., 2002, 10, (3), pp. 381-392

14 Tanelli, M., Piroddi, L., Savaresi, S.M.: 'Real-time identification of tire-road friction conditions', IET Control Theory Appl., 2009, 3, (7), pp. 891-906

15 Hori, Y.: 'Future vehicle driven by electricity and control - research on four-wheel-motored UOT Electric March II', IEEE Trans. Ind. Electron., 2004, 51, (5), pp. 954-962
16 Pacejka, H.B.: 'Tyre and vehicle dynamics' (Butterworth-Heinemann, 2002)

17 Pasillas-Lepine, W.: 'Hybrid modeling and limit cycle analysis for a class of five-phase anti-lock brake algorithms', Veh. Syst. Dyn., 2006, 44, (2), pp. 173-188

18 de Castro, R., Araujo, R.E., Cardoso, J.S., Freitas D.: 'A new linear parametrization for peak friction coefficient estimation in real time', IEEE Vehicle Power and Propulsion Conf. (VPPC), Lille, France, 2010 .

19 Alvarez, L., Jingang, Y.: 'Adaptive emergency braking control in automated highway systems'. Proc. 38th IEEE Conf. on Decision and Control, 1999, 4, pp. 3740-3745

20 Chia, T.L., Chow, P.C., Chizeck, H.J.: 'Recursive parameter identification of constrained systems: an application to electrically stimulated muscle', IEEE Trans. Biomed. Eng., 1991, 38, (5), pp. $429-442$

21 Resende, L.S., Romano, J.M.T., Bellanger, M.G.: 'A fast least-squares algorithm for linearly constrained adaptive filtering', IEEE Trans. Signal Process., 1996, 44, (5), pp. 1168-1174

22 Canudas-de Wit, C., Tsiotras, P., Velenis, E., Basset M., Gissinger, G.: 'Dynamic friction models for road/tire longitudinal interaction', Veh. Syst. Dyn., 2003, 39, (3), pp. 189-226

23 Li, L., Wang F.-Y.: 'Advanced motion control and sensing for intelligent vehicles' (Springer Verlag, 2007)

24 Corno, M., Gerard, M., Verhaegen, M., Holweg, E.: 'Hybrid ABS control using force measurement', IEEE Trans. Control Syst. Technol., 2012, 20, (5), pp. 1223-1235.

25 Leeuwen, B.V., Zuurbier, J.: 'Vehicle state estimation based on load sensing'. Veh. Dyn. Expo, 2007, Germany.

26 Savaresi, S.M., Tanelli, M., Langthaler, P., Del Re, L.: 'New regressors for the direct identification of tire deformation in road vehicles via in-tire accelerometers', IEEE Trans. Control Syst. Technol., 2008, 16, (4), pp. 769-780

27 Hori, Y., Toyoda, Y., Tsuruoka, Y.: 'Traction control of electric vehicle: basic experimental results using the test EV "UOT Electric March",' IEEE Trans. Ind. Appl., 1998, 34, (5), pp. 1131-1138

28 Baffet, G., Charara, A., Dherbomez, G.: 'An observer of tire-road forces and friction for active security vehicle systems', IEEE/ASME Trans. Mechatronics, 2007, 12, (6), pp. 651-661

29 Ray, L.R.: 'Nonlinear tire force estimation and road friction identification: simulation and experiments', Automatica, 1997, 33, (10), pp. 1819-1833

30 van Zanten, A., Erhardt, R., Lutz, A.: 'Measurement and simulation of transients in longitudinal and lateral tire forces', SAE Trans., 1990 99, (6), pp. 300-318

31 Ziena Optimization I.: 'KNITRO 6.0 User Manual', 2009

32 TMPT, 'Tyre Model Performance Test', 2003, available at: http://tmpt.tuwien.ac.at/

33 Ljung, L.: 'System identification: theory for the user' (Prentice-Hall, 1999)

34 Rao, C., Toutenburg, H., Shalabh, Heumann, C.: 'Linear models and generalizations: least squares and alternatives' (Springer Verlag, 2008, 3rd edn.)

35 Isermann, R., Munchhof, M.: 'Identification of dynamic systems: an introduction with applications' (Springer-Verlag, 2011)

36 Alenany, A., Shang, H., Soliman, M., Ziedan, I.: 'Improved subspace identification with prior information using constrained least squares', IET Control Theory Appl., 2011, 5, (13), pp. 1568-1576

37 Aguirre, L.A., Barroso, M.F.S., Saldanha, R.R., Mendes E.M.A.M.: 'Imposing steady-state performance on identified nonlinear polynomial models by means of constrained parameter estimation', IEE Proc. Control Theory Appl., 2004, 151, (2), pp. 174-179

38 'CarSim 8.0 User Manual', 2009.

39 de Castro, R., Araujo, R.E., Freitas, D.: 'Reusable IP cores library for EV propulsion systems' IEEE Int. Symp. on Industrial Electronics, Bari, Italy, 2010.

40 Araujo, R.E., Ribeiro, G., de Castro, R.P., Oliveira H.S.: 'Experimental evaluation of a loss-minimization control of induction motors used in EV', Thirty-fourth Annual Conf. on IEEE Industrial Electronics, Orlando, FL, 2008, pp. 1194-1199.

41 de Castro, R., Araujo, R.E., Freitas, D.: 'A single motion chip for multi-motor EV control', Tenth Int. Symp. on Advanced Vehicle Control (AVEC), Loughborough, UK, 2010.

42 Stotsky, A. Kolmanovsky, I.: 'Application of input estimation techniques to charge estimation and control in automotive engines', Control Eng. Pract., 2002, 10, (12), pp. 1371-1383

43 Luenberger, D.G.: 'Optimization by vector space methods' (WileyIEEE, 1969) 


\section{www.ietdl.org}

\section{Appendix 1. Classic approximation result}

This section reviews a simple result from the theory of function approximation, which is helpful in establishing the optimal LP. For this purpose, consider the problem of approximating (6) with (7), assuming that $\beta$ and $\boldsymbol{w}$ are fixed and known. Our intention is to find the linear parameters $\boldsymbol{\theta}$, such that the fitting performance, defined as the integral of the square fitting error $(\varepsilon)$, is minimised

$$
\min _{\boldsymbol{\theta} \in \mathbb{R}^{n}} \varepsilon(\boldsymbol{\theta})=\min _{\boldsymbol{\theta} \in \mathbb{R}^{n}} \int_{0}^{\bar{\lambda}}(f(\lambda)-\underbrace{\boldsymbol{H}(\lambda)^{\mathrm{T}} \boldsymbol{\theta}}_{\hat{f}(\lambda, \boldsymbol{\theta})})^{2} \mathrm{~d} \lambda
$$

Note that, to simplify the notation, the dependence of $\beta$ in $f$ (and of $\boldsymbol{w}$ in $\boldsymbol{H}$ ) is omitted in this section, which is reasonable as these parameters are assumed to be constant. The previous optimisation problem can be formulated in the vector space $\left.L_{2}([0, \bar{\lambda}], \mathbb{R}]\right)$, that is, square-integrable functions over the interval $[0, \bar{\lambda}]$, making use of the $L_{2}$ norm

$$
\varepsilon(\boldsymbol{\theta})=\|f-\hat{f}\|^{2}=\int_{0}^{\bar{\lambda}}(f(\lambda)-\hat{f}(\lambda, \boldsymbol{\theta}))^{2} \mathrm{~d} \lambda
$$

Since the vector $\hat{f}$ is a linear combination of $L_{2}$ vectors [cf. (7)], the optimisation problem (35) can be reformulated as a minimum norm problem in the $L_{2}$ space, which has a simple analytical solution, as described in the following lemma.
Lemma 1: Consider the vector space $\left.L_{2}([0, \bar{\lambda}], \mathbb{R}]\right)$, a vector $f \in L_{2}$ and a subspace $\mathcal{M} \subset L_{2}$ generated by the linear combination of $n$ basis functions $h_{i} \in L_{2}$

$$
\mathcal{M}=\left\{\hat{f} \in L_{2}: \hat{f}=\sum_{i=1}^{n} h_{i} \theta_{i}, \quad h_{i} \in L_{2}, \quad \theta_{i} \in \mathbb{R}\right\}
$$

Then, the minimum norm problem

$$
\min _{\hat{f} \in \mathcal{M}}\|f-\hat{f}\|^{2}
$$

has the following solution

$$
\begin{array}{r}
\hat{f}_{\mathrm{o}}(\lambda)=\left[h_{1}(\lambda), \ldots, h_{n}(\lambda)\right] \boldsymbol{\theta}_{\mathbf{0}}, \quad \boldsymbol{\theta}_{\mathbf{0}}=\boldsymbol{G}^{-1} \boldsymbol{c} \\
{[\boldsymbol{G}]_{i, j}=\left\langle h_{i}, h_{j}\right\rangle=\int_{0}^{\bar{\lambda}} h_{i}(\lambda) h_{j}(\lambda) \mathrm{d} \lambda} \\
{[\boldsymbol{c}]_{i}=\left\langle f, h_{i}\right\rangle=\int_{0}^{\bar{\lambda}} f(\lambda) h_{i}(\lambda) \mathrm{d} \lambda}
\end{array}
$$

where $i, j=1, \ldots, n, \boldsymbol{G} \in \mathbb{R}^{n \times n}, \boldsymbol{c} \in \mathbb{R}^{n},\langle$,$\rangle is the L_{2}$ inner product, and $[.]_{i, j}$ refers to row $i$, column $j$ of a given matrix. Moreover, the solution is unique if the basis functions $h_{i}$ are linearly independent.

Proof: Given the fact that $L_{2}$ is a Hilbert space, we can apply the projection theorem to solve this minimum norm problem (see [43, Chapter 3.6] for details). 